\title{
Measuring serum estradiol and progesterone one day prior to frozen embryo transfer improves live birth rates
}

\author{
Snigdha Alur-Gupta ${ }^{1 *}$, Margaret Hopeman ${ }^{1,2}$, Dara S. Berger ${ }^{1}$, Kurt T. Barnhart ${ }^{1}$, Suneeta Senapati ${ }^{1}$ and
} Clarisa Gracia ${ }^{1}$

\begin{abstract}
Background: Given no consensus in the literature, this study sought to determine if a protocol of measuring serum estradiol and progesterone the day prior to frozen embryo transfer (FET) improves likelihood of pregnancy and livebirth.

Methods: This was a retrospective time-series study of women undergoing autologous vitrified-warmed blastocyst programmed FETs at an academic institution. Live birth rates were compared between a surveillance protocol, where serum estrogen and progesterone surveillance are performed the day prior to a programmed FET, and a standard protocol, whereby no hormonal lab evaluation is performed the day prior.

Results: Three hundred seventy-nine standard FET and 524 surveillance FET cycles were performed. Patients in the surveillance protocol were significantly more likely to achieve live birth (51\% vs. 39\%; aOR 1.6, 95\%Cl [1.2, 2.2]). Obese women were noted to be more likely to have lower progesterone hormone levels on surveillance labs (OR $3.2,95 \% \mathrm{Cl}[2.0,5.3])$. However those whose hormonal medication dose was modified because of pre-transfer labs were as likely to achieve live birth as those whose dose was not modified (47\% vs. 53\%; aOR 0.8, 95\% Cl [0.6, 1.2]).

Conclusions: Cycles with the surveillance protocol were more likely to result in live birth. Patients with low levels of pre-transfer hormones, such as obese patients, likely have lower pregnancy rates. It is possible that when these levels were corrected after measurement, pregnancy rates improved to match those whose levels were not low enough to warrant intervention.
\end{abstract}

Keywords: Assisted reproductive technology, Frozen embryo transfer, Hormone surveillance, Live birth, Pregnancy, Progesterone level

\section{Background}

Frozen embryo transfers (FETs) have increased in prevalence, with autologous frozen embryo cycles accounting for $32.7 \%$ of all ART cycles conducted in the United States in 2016 [1]. FETs are performed not only to

\footnotetext{
* Correspondence: snigdha.alur-gupta@pennmedicine.upenn.edu

'Division of Reproductive Endocrinology and Infertility, University of Pennsylvania, Philadelphia, Pennsylvania, USA

Full list of author information is available at the end of the article
}

utilize supernumerary embryos but are recommended for women with an inadequate endometrium, or those pursuing preimplantation genetic testing (PGT) [2, 3]. As use increases, it is important to identify factors that improve outcomes [4]. Several known predictors include age and number and quality of frozen embryos [4]. Another vital aspect is the 'window of implantation', the time period when the endometrium is most receptive [3, 5]. This state is induced through an interplay of hormones including estrogen and progesterone [6]. Prior

(c) The Author(s). 2020 Open Access This article is licensed under a Creative Commons Attribution 4.0 International License, which permits use, sharing, adaptation, distribution and reproduction in any medium or format, as long as you give appropriate credit to the original author(s) and the source, provide a link to the Creative Commons licence, and indicate if changes were made. The images or other third party material in this article are included in the article's Creative Commons licence, unless indicated otherwise in a credit line to the material. If material is not included in the article's Creative Commons licence and your intended use is not permitted by statutory regulation or exceeds the permitted use, you will need to obtain permission directly from the copyright holder. To view a copy of this licence, visit http://creativecommons.org/licenses/by/4.0/ The Creative Commons Public Domain Dedication waiver (http://creativecommons.org/publicdomain/zero/1.0/) applies to the data made available in this article, unless otherwise stated in a credit line to the data. 
evidence has shown that premature elevations in progesterone alter this window and can be associated with lower pregnancy rates in fresh cycles [7-9].

Therefore, when performing FETs an attempt is made to replicate the natural hormonal environment. While extensive research has evaluated ideal protocols for endometrial preparation, no definitive method has been established [10-12]. The optimal route of progesterone or estradiol administration [13-15] and duration of progesterone administration $[16,17]$ have all been studied. While intramuscular progesterone has been shown to be superior to Endometrin for ongoing pregnancy rate in an interim analysis of a large randomized non-inferiority study [15], significant differences in implantation, clinical pregnancy and live birth rates were not observed in a retrospective evaluation comparing vaginal progesterone gel to intramuscular progesterone [14]. It is also unclear whether hormone levels surrounding time of frozen transfer are associated with outcome. In a review of women undergoing autologous euploid embryo transfers with intramuscular progesterone supplementation, day 19 progesterone levels above $20 \mathrm{ng} / \mathrm{mL}$ were noted to be associated with lower live birth rates, with more drastic decreases as progesterone levels crossed $40 \mathrm{ng} / \mathrm{ml}$ [18]. However a separate study assessing fresh untested day 3 donor embryo transfers with intramuscular progesterone supplementation found that progesterone levels above $20 \mathrm{ng} / \mathrm{mL}$ were instead associated with higher live birth rates [19]. A study of autologous euploid frozen embryo transfers with vaginal progesterone support found that women in the lower quartiles of serum progesterone levels $(<10.64 \mathrm{ng} / \mathrm{mL})$ the day prior to transfer had significantly higher miscarriage and lower live birth rates compared to those in the other quartiles [20]. A similarly structured prospective cohort study of both fresh and frozen donor oocyte transfer cycles with vaginal progesterone support found that women with progesterone levels under $9.2 \mathrm{ng} / \mathrm{mL}$ had significantly lower adjusted ongoing pregnancy rates compared to those with levels above this threshold [21]. However, both these studies used vaginal progesterone supplementation which was shown to be inferior as described above and makes interpretation of serum levels more difficult. The varying patient populations, use of autologous versus donor oocytes, evaluation of both fresh and frozen cycles and differing routes of progesterone administration in all of these studies make it challenging to draw conclusions.

Given the conflicting information and the biologic plausibility by which these hormonal measurements are important, we sought to study whether a protocol of monitoring pre-transfer estradiol and progesterone levels and adjusting hormone doses impacted pregnancy and live birth rates in a well-defined modern cohort of women undergoing autologous frozen embryo transfers with intramuscular progesterone supplementation.

\section{Material and methods}

This was a single center retrospective time-series study conducted from January 2013-February 2017. Reproductive aged women undergoing programmed autologous vitrified-warmed blastocyst transfers, referred to as FETs, were included. Our laboratory uses vitrification and thaw media manufactured by Fuji Film Irvine Scientific (Santa Ana, CA). Embryos are frozen and stored on CBS High Security Vitrification straws manufactured by Cryo Bio System (L'Aigle, France). We converted from a slow freezing protocol to vitrification during the third quarter of 2012, before this study inclusion period. Some of the earlier embryos may have been frozen by the original slow freezing protocol, however still thawed using Irvine Scientific media, per laboratory protocol. Those using donor oocytes, gestational carriers or natural cycle FETs were excluded. Patients of all ages and fertility diagnoses were included. Patients who had FETs performed between January 2013 and June 2015 received the standard FET protocol, described in further detail below and referred to as the "standard protocol". Beginning in June 2015, serum estradiol and progesterone levels were obtained the day prior to transfer in order to determine if hormonal medication dose adjustments were required prior to the FET. Those who had FETs performed after June 2015 thus received the standard protocol with the addition of the pre-transfer lab surveillance, referred to as the "surveillance protocol", reviewed below.

\section{Standard protocol}

A FET was performed by first administering luteal phase $\mathrm{GnRH}$ agonist suppression for a minimum of 12 days. Ovarian suppression was confirmed with baseline hormonal and transvaginal ultrasound assessment. If confirmed to be suppressed, oral estradiol was initiated at a dose of $2 \mathrm{mg}$ and titrated to $6 \mathrm{mg}$ daily over the course of 12 days. Transvaginal ultrasound and bloodwork was performed after these 12 days and embryo transfer was scheduled if the endometrial thickness was at least $7 \mathrm{~mm}$ and of a trilaminar morphology and estradiol levels at least $200 \mathrm{pg} / \mathrm{mL}$. If progesterone levels were greater than or equal to $1.5 \mathrm{ng} / \mathrm{ml}$, the cycle was cancelled with plan for higher doses of $\mathrm{GnRH}$ agonist in subsequent cycles. In cases of either inadequate estradiol level, endometrial thickness or morphology, typically vaginal estradiol at a dose of 1-2 mg or higher doses of oral estradiol (increased by increments of $2 \mathrm{mg}$ ) were administered for approximately 1 week and bloodwork and transvaginal ultrasound were repeated to assess if above specified criteria were met. Intramuscular progesterone was initiated 
at $50 \mathrm{mg}$ nightly when parameters were met, and blastocyst transfer was scheduled to occur on the 6th day of progesterone supplementation.

\section{Surveillance protocol}

Endometrial preparation followed the same methods as outlined above. However, patients undergoing FETs after June 2015 also had serum estradiol and progesterone surveillance the day prior to their FET, on the 5th day of progesterone supplementation. Estradiol doses were increased by 1-2 mg vaginally or orally by $2 \mathrm{mg}$ for levels below $150 \mathrm{pg} / \mathrm{mL}$. Progesterone was typically increased from $50 \mathrm{mg}$ to $75 \mathrm{mg}$ IM nightly, if levels were below 15 $\mathrm{ng} / \mathrm{mL}$. These thresholds were established based upon clinical consensus within the center, given that there are no uniform threshold recommendations based upon prior research. Transfers in both protocols were cancelled for inadequate endometrial lining or morphology or inappropriate estradiol levels.

\section{Outcome assessment}

Serum hCG was performed 10-12 days following transfer. bHCGs $>1 \mathrm{mIU} / \mathrm{mL}$ were considered positive and used to define pregnancy rate. Primary outcomes were pregnancy rate and live birth rates. The following definitions were employed for secondary outcomes: implantation rate: number of fetal sacs divided by number of embryos transferred, biochemical pregnancy: positive hCG that spontaneously dropped to $<1 \mathrm{mIU} / \mathrm{mL}$ in the absence of an intrauterine gestational sac; clinical intrauterine pregnancy: presence of an intrauterine gestational and yolk sac on ultrasound; spontaneous abortion: loss of clinical intrauterine pregnancy; therapeutic abortion: induced loss of clinical intrauterine pregnancy and stillbirth: pregnancy losses at greater than 20 weeks gestation.

\section{Analysis}

In order to have $80 \%$ power to detect $10 \%$ increase in pregnancy rates between the two groups, 380 cycles per group were needed. Categorical data was analyzed using chi-square test whereas continuous data was analyzed by either student t-test or Wilcoxson ranksum as indicated by normality assessment. Multivariate logistic and linear regression was used to compare primary and secondary outcomes, adjusting for age, body mass index (BMI), usage of PGT, and number of embryos transferred. Confounders were determined apriori as well as through a backwards elimination strategy.

Multivariable analysis was first conducted to compare primary and second outcomes for women in the surveillance protocol versus those in the standard protocol. In a sub-analysis, women in the surveillance protocol who had hormonal medication dose adjustment as a result of surveillance labs were then compared to those in the surveillance protocol whose doses did not require adjustment. Sensitivity analyses were conducted to evaluate differences in live birth rates between the surveillance and standard protocols for those who had freeze-all cycles (defined as those who did not have a fresh embryo transfer at the time of stimulation) and those who did and did not have PGT. These specific groups were chosen as patients with freeze-all cycles and PGT are thought to be better prognosis patients which could influence primary outcome results. Analysis was performed using STATA v.14.

\section{Results}

Baseline characteristics: surveillance versus standard protocol

Three hundred seventy-nine FETs were performed with the standard protocol and 524 were performed with the surveillance protocol. Cancellations, which occurred in $4.1 \%$ of standard and $5.8 \%$ of surveillance protocol cycles, were most often due to structural factors such as thin endometrial lining $(<7 \mathrm{~mm})$. The mean age and BMI of women did not differ significantly in the two groups. Those in the standard protocol group were significantly less likely to have PGT and more likely to have more embryos transferred and have multiple gestations (defined by number of heartbeats) $(p<0.001)$. (See Table 1).

\section{Surveillance versus standard protocol}

In multivariable logistic regression analyses, patients undergoing FETs with the surveillance protocol were significantly more likely to become pregnant $(70 \%$ vs 61\%; aOR 1.6, 95\% CI $[1.2,2.2])$ as well as achieve live birth (51\% vs. $39 \%$; aOR $1.6,95 \% \mathrm{CI}[1.2,2.2])$ compared to those in the standard protocol. (See Table 3 for logistic regression results). In multivariable linear regression models, patients undergoing FETs with the surveillance protocol also had a significantly higher implantation rate $(p=0.04)$. (Not shown).

Analyses restricted to freeze-all cycles were similar when comparing live birth rates between the surveillance and standard protocols (aOR 1.6, 95\% CI [1.1, 2.5]). We also performed sensitivity analyses stratified by PGT. In patients who did not have PGT, the odds of live birth in the surveillance protocol was still increased (aOR 1.6 $95 \% \mathrm{CI}[1.2,2.3])$. In the population of those who underwent PGT, the odds of live birth in the surveillance protocol was similarly increased (aOR 1.5 95\% CI [0.8, $2.8]$ ), however it did not reach statistical significance. Given the possibility that women may have had embryos frozen via the slow-freeze method prior to transfer during the study period, an additional restricted analysis was performed removing all women with embryo 
Table 1 Baseline Characteristics of Patients in Surveillance vs Standard Protocols

\begin{tabular}{|c|c|c|c|}
\hline & Surveillance protocol $(\boldsymbol{N}=524)$ & Standard protocol $(\boldsymbol{N}=379)$ & $\boldsymbol{p}$-value \\
\hline Mean Age (years) & $34.6( \pm 4.3)$ & $34.7( \pm 3.9)$ & 0.7 \\
\hline Mean BMI $\left(\mathrm{kg} / \mathrm{m}^{2}\right)$ & $25.3( \pm 5.3)$ & $25.0( \pm 5.3)$ & 0.13 \\
\hline \multirow[t]{6}{*}{ Infertility Diagnosis (\%) } & Unexplained - (25\%) & Unexplained - (23\%) & 0.1 \\
\hline & DOR - (6\%) & DOR - (8\%) & \\
\hline & Endometriosis - (3\%) & Endometriosis - (8\%) & \\
\hline & Ovulation disorders - (11\%) & Ovulation disorders - (10\%) & \\
\hline & Male factor - (21\%) & Male factor - (19\%) & \\
\hline & Other $^{\mathrm{a}}-(34 \%)$ & Other $^{a}-(32 \%)$ & \\
\hline PGT testing of embryo, $n$ (\%) & $167 / 524(32 \%)$ & $52 / 379(14 \%)$ & $<0.001$ \\
\hline \multirow[t]{3}{*}{ Number of embryos transferred per cycle, $n(\%)$} & $1-351(67 \%)$ & $1-194(51 \%)$ & $<0.001$ \\
\hline & $2-168(32 \%)$ & $2-174(46 \%)$ & \\
\hline & $3-5(1 \%)$ & $3-10(3 \%)$ & \\
\hline \multirow[t]{3}{*}{ Number of heartbeats, $n$ (\%) } & $1-245(76 \%)$ & $1-135(80 \%)$ & $<0.001$ \\
\hline & $2-41(13 \%)$ & $2-33(19 \%)$ & \\
\hline & $3-4(1 \%)$ & $3-0(0 \%)$ & \\
\hline
\end{tabular}

${ }^{a}=$ Included patients with tubal factor, uterine factor and other as infertility diagnoses

cryopreservation performed prior to adaption of the vitrification protocol. In total, 38 women were removed and results for both primary outcomes of pregnancy and live birth remained unchanged.

\section{Baseline characteristics: those with versus without dose adjustments in surveillance protocol}

Women in the surveillance protocol whose hormone doses were adjusted based on surveillance labs were not significantly different from those without dose adjustment in terms of age, endometrial thickness, infertility diagnosis, use of PGT or number of embryos transferred. Mean BMI was significantly higher in the dose adjusted group compared to the dose unadjusted group. $(p<$ 0.001) (See Table 2) During the surveillance protocol time period, progesterone dosage was increased in $26 \%$ of cycles, estradiol in 11\%, and both in 33\%. Obese women were more likely to have lower progesterone levels requiring dose adjustment (OR 3.2, 95\% CI [2.0, 5.3]). Of the women who underwent PGT in the surveillance protocol, $28 \%$ required hormone dose changes and $72 \%$ did not.

\section{Dose adjusted versus dose unadjusted cycles in surveillance protocol}

In multivariable logistic regression analyses, those whose dose was adjusted in the surveillance protocol group were as likely to become pregnant as those whose dose did not need to be adjusted ( $72 \%$ vs $66 \%$; aOR $0.8,95 \%$ CI $[0.5,1.2])$. They were also as likely to achieve live birth compared to those whose dose was unadjusted (47\% vs. 53\%; aOR 0.8, 95\%CI $[0.6,1.2]$ ) (See Table 3 for logistic regression results). Sensitivity analyses restricted to either solely estrogen dose changes or progesterone dose changes did not change livebirth results substantially (aOR $0.6,95 \% \mathrm{CI}[0.4,1.2]$ and aOR $0.9,95 \% \mathrm{CI}$ $[0.6,1.4]$ respectively) when compared to the overall results in which either medication was changed. In multivariable linear regression models, patients undergoing FETs with dose adjustment were as likely to implant as those without dose adjustment ( $p=0.3)$. (Not shown).

\section{Discussion}

We found that pregnancy and live birth rates were significantly higher when implementing the surveillance protocol as compared to the standard protocol. The number of studies evaluating the impact of surveillance blood work on outcomes is limited. Unlike our findings, other studies have not demonstrated a consistent association between pre-transfer serum hormone levels and outcome, with one noting that differences in estradiol concentrations prior to FET did not correlate with clinical pregnancy rates [22]. While we did not have a group of patients with surveillance revealing low estradiol levels but no dose adjustment to compare to (as all patients in the surveillance protocol with inadequate levels had dose adjustments), comparison with patients in the standard protocol would indicate that assessment appears to impact outcome.

Interestingly however, women whose hormone doses were adjusted based on the surveillance labs were as likely to conceive as women whose doses were unadjusted. Although it seems counterintuitive that dose adjustment did not improve rates, we believe this may 
Table 2 Baseline Characteristics of those whose treatment was changed due to pre-transfer surveillance labs versus those where labs were measured but treatment was not changed

\begin{tabular}{|c|c|c|c|}
\hline & $\begin{array}{l}\text { Patients with change in } \\
\text { hormones }(\boldsymbol{N}=175)\end{array}$ & $\begin{array}{l}\text { Patients without change in } \\
\text { hormones }(\boldsymbol{N}=349)\end{array}$ & $p$-value \\
\hline Mean Age (years) & $34.2( \pm 4.8)$ & $34.8( \pm 4.1)$ & 0.2 \\
\hline Mean BMI $\left(\mathrm{kg} / \mathrm{m}^{2}\right)$ & $26.8( \pm 5.8)$ & $23.6( \pm 4.8)$ & $<0.001$ \\
\hline Endometrial stripe (mm) & $10( \pm 2.4)$ & $9.6( \pm 2.1)$ & 0.06 \\
\hline \multirow[t]{6}{*}{ Infertility Diagnosis (\%) } & Unexplained - (23\%) & Unexplained - (27\%) & 0.3 \\
\hline & DOR - (3\%) & $\mathrm{DOR}-(7 \%)$ & \\
\hline & Endometriosis - (5\%) & Endometriosis - (3\%) & \\
\hline & Ovulation disorders - (11\%) & Ovulation disorders - (10\%) & \\
\hline & Male factor - $(25 \%)$ & Male factor - (19\%) & \\
\hline & Other $^{a}-(33 \%)$ & Other $^{\mathrm{a}}-(34 \%)$ & \\
\hline \multirow[t]{3}{*}{ Number of embryos transferred per cycle, $n(\%)$} & $1-118(67.4 \%)$ & $1-233(67 \%)$ & 0.8 \\
\hline & $2-56(32 \%)$ & $2-112(32 \%)$ & \\
\hline & $3-1(0.6 \%)$ & $3-4(1 \%)$ & \\
\hline PGT testing of embryo, $n$ (\%) & $46 / 175(26 \%)$ & $121 / 349(35 \%)$ & 0.05 \\
\hline
\end{tabular}

$\mathrm{a}=$ Included patients with tubal factor, uterine factor and other as infertility diagnoses

be related to group characteristics. The group requiring dose adjustment may represent poorer prognosis patients. Interestingly, the mean BMI of women in this group was in the overweight range (mean BMI $26.8 \mathrm{~kg}$ / $\mathrm{m}^{2}$ ) compared to the normal BMI in the unadjusted dose group (mean BMI $23.6 \mathrm{~kg} / \mathrm{m}^{2}$ ). In addition, $50.6 \%$ of women in the dose adjusted group were overweight or obese compared to only $36.6 \%$ in the dose unadjusted group. Factors such as obesity have been associated with poorer outcomes [23-26]. It is possible that by adjusting hormone doses, we were able to increase this group's pregnancy rates to be similar to normal weight, better prognosis patients in the comparison cohort. However, since there was no untreated control group with hormone level monitoring, we acknowledge it is not possible to determine whether this intervention was responsible for the rise in pregnancy rates or by how much. In addition, a sensitivity analysis evaluating pregnancy and live births restricted to non-obese women in the hormone adjusted group versus not-adjusted group within the surveillance protocol cohort also did not show differences in outcomes (not shown). However, as this was not the primary objective, it is difficult to know whether this is an issue of power or if alternative explanations are necessary.

During the timeframe of the standard protocol, PGT was not as common and embryo transfer guidelines were less stringent $[27,28]$. Therefore, it is not surprising that women in this group were significantly different with respect to these parameters. In the final multivariate analysis, we adjusted for PGT and number of embryos transferred to account for these differences. It is less likely that results shown are significantly affected by differences in PGT alone as sensitivity analyses yielded similar findings, with the non-significant results likely related to the smaller size of the PGT group.

Table 3 Pregnancy Outcome of Patients in Surveillance vs Standard protocols \& Change in Hormones vs No Change

\begin{tabular}{|c|c|c|c|c|c|c|c|c|}
\hline & $\begin{array}{l}\text { Surveillance } \\
\text { protocol } \\
(\boldsymbol{N}=524)\end{array}$ & $\begin{array}{l}\text { Standard } \\
\text { protocol } \\
(\boldsymbol{N}=379)\end{array}$ & $\begin{array}{l}\text { aOR } \\
(95 \% \mathrm{Cl})\end{array}$ & $\boldsymbol{p}$-value & $\begin{array}{l}\text { Patients with } \\
\text { change in } \\
\text { hormones } \\
(\boldsymbol{N}=175)\end{array}$ & $\begin{array}{l}\text { Patients without } \\
\text { change in } \\
\text { hormones } \\
(\boldsymbol{N}=349)\end{array}$ & $\begin{array}{l}\text { aOR } \\
(95 \% \mathrm{Cl})\end{array}$ & $\bar{p}$-value \\
\hline Positive hCG $^{\text {a }}$ & $366 / 524(70 \%)$ & $231 / 379(61 \%)$ & $1.6(1.2,2.2)$ & 0.002 & $115 / 175(66 \%)$ & $251 / 349(72 \%)$ & $0.8(0.5,1.2)$ & 0.3 \\
\hline Live birth & $268(51 \%)$ & 149 (39\%) & $1.6(1.2,2.2)$ & 0.001 & $83(47 \%)$ & $185(53 \%)$ & $0.8(0.6,1.2)$ & 0.4 \\
\hline Spontaneous abortion & $51(10 \%)$ & $38(10 \%)$ & $1.0(0.7,1.7)$ & 0.9 & $13(7 \%)$ & $38(11 \%)$ & $0.6(0.3,1.2)$ & 0.2 \\
\hline Therapeutic abortion & $4(0.8 \%)$ & $2(0.5 \%)$ & $1.4(0.2,8.1)$ & 0.7 & $1(0.6 \%)$ & $3(0.9 \%)$ & $0.7(0.1,7.4)$ & 0.8 \\
\hline Stillborn (> 20 weeks) & $2(0.4 \%)$ & $2(0.5 \%)$ & $0.9(0.1,6.6)$ & 0.9 & $1(0.6 \%)$ & $1(0.3 \%)$ & $2.0(0.1,35.0)$ & 0.6 \\
\hline Ectopic Pregnancy & $1(0.2 \%)$ & $2(0.5 \%)$ & $0.5(0.1,6.2)$ & 0.6 & $0(0 \%)$ & $1(0.3 \%)$ & - & - \\
\hline Biochemical Pregnancy & $40(7.6 \%)$ & $38(10 \%)$ & $0.8(0.5,1.3)$ & 0.4 & $17(10 \%)$ & $23(7 \%)$ & $1.7(0.9,3.4)$ & 0.1 \\
\hline
\end{tabular}

All analyses were performed using logistic regression adjusted for age, BMI, number of embryos transferred and PGT

${ }^{a}=$ Positive hCG represents summation of all pregnancy outcomes listed below this row 
Our study has several notable strengths. We were able to perform a large retrospective study of women representative of the modern infertile population. Our results remained robust in analyses restricted to freeze-all cycles which generally represent better prognostic patients. However, there are some limitations owing to the retrospective nature. We did not have a group of patients who had pre-transfer lab assessment indicating suboptimal levels but without dose adjustment. Therefore, it is not possible to directly assess how pregnancy rates were influenced by adjusting doses. While it is possible for changes in lab practices between 2013 and 2017 to influence outcomes, overall our management has remained similar, including methods of IVF stimulation and embryo transfer procedure that would most directly impact outcome. For example, the brand of transfer catheters did not change, the catheter loading process remained consistent and the culture system in the laboratory as well as general embryology techniques were not altered. While the gas delivery system to incubators was upgraded from Teflon tubing to copper pipping in July 2016, thawed embryos remain in culture for only approximately $2 \mathrm{~h}$ prior to embryo transfer; therefore, we do not feel this would have a significant impact on FET outcomes. In addition, the two cohorts were similar in several pertinent ways including age, BMI and infertility diagnoses. Factors that would have changed with time in laboratory practices, such as prevalence of PGT and number of embryos transferred, were controlled for in all models. In addition, restricted analyses removing those with embryos frozen via the slow-freeze method also showed the same results. We did not evaluate hormone levels after FETs to assess whether levels were still suboptimal; therefore, it is possible that inadequate dose adjustment could contribute to lack of differences. Embryo morphology data was also not analyzed.

In conclusion, we found that pregnancy and live birth rates significantly improved after implementing the surveillance protocol, however rates were not significantly different in those whose dose was adjusted based on surveillance labs compared to those whose dose was not adjusted. We hypothesize that patients with low levels on pre-transfer labs may represent a poor prognosis group, such as obese individuals, and we were able to improve their pregnancy rates to match those who did not require hormonal medication dose adjustment by hormonal testing and subsequent medication adjustment. Given the increased prevalence of FETs, understanding how serum evaluation influences outcome is essential. Based on our findings, pre-transfer or day of transfer serum evaluation is warranted and it is possible that obese women should be prescribed higher doses of progesterone in order to prevent suboptimal levels on pretransfer blood work. However, data on appropriate titration of intramuscular progesterone by BMI is lacking. Performing blood work the day of transfer immediately prior to transfer may decrease repetitive hospital visits. Prospective randomized control trials are needed to explore this area further.

\section{Acknowledgements \\ We would like to thank BabySentry for their assistance with querying the database.}

\section{Authors' contributions}

SAG contributed to the design of the work, the acquisition, analysis, interpretation of data, drafting and revising the manuscript. $\mathrm{MH}$ contributed to the design of the work, analysis and interpretation of data. DS contributed to acquisition of data and revising the manuscript. KB contributed to design of the work, interpretation of data and revising the manuscript. SS

contributed to analysis, interpretation of data and revising the manuscript.

CG contributed to the design of the work, interpretation of data and revising the manuscript. All authors read and approved the final manuscript.

\section{Funding}

Snigdha Alur-Gupta and Margaret Hopeman are supported by the National Institute of Health under T32 Training Grant: HD007440. This source did not play any role in the design of the study, collection, analysis and interpretation of data or in writing the manuscript.

\section{Availability of data and materials}

All data generated or analyzed during this study are included in this published article.

Ethics approval and consent to participate

Approval for the study was obtained from the University of Pennsylvania's Institutional Review Board.

Consent for publication

Not applicable.

\section{Competing interests}

The authors declare that they have no competing interests.

\section{Author details}

${ }^{1}$ Division of Reproductive Endocrinology and Infertility, University of Pennsylvania, Philadelphia, Pennsylvania, USA. ${ }^{2}$ Present address: Center for Reproductive Medicine \& Advanced Reproductive Technologies, Twin Cities, Minnesota, USA.

Received: 2 May 2019 Accepted: 30 March 2020

Published online: 14 April 2020

\section{References}

1. 2016 National Summary Report. In: National Center for Chronic Disease Prevention and Health Promotion DoRH. 2016.

2. Shapiro BS, Daneshmand ST, Garner FC, Aguirre M, Hudson C. Clinical rationale for cryopreservation of entire embryo cohorts in lieu of fresh transfer. Fertil Steril. 2014;102(1):3-9.

3. Casper RF, Yanushpolsky EH. Optimal endometrial preparation for frozen embryo transfer cycles: window of implantation and progesterone support. Fertil Steril. 2016;105(4):867-72.

4. Veleva Z, Orava M, Nuojua-Huttunen S, Tapanainen JS, Martikainen H. Factors affecting the outcome of frozen-thawed embryo transfer. Hum Reprod. 2013;28(9):2425-31.

5. Bergh PA, Navot D. The impact of embryonic development and endometrial maturity on the timing of implantation. Fertil Steril. 1992;58(3): 537-42.

6. Simon C, Martin JC, Pellicer A. Paracrine regulators of implantation. Baillieres Best Pract Res Clin Obstet Gynaecol. 2000;14(5):815-26.

7. Lawrenz B, Fatemi HM. Effect of progesterone elevation in follicular phase of IVF-cycles on the endometrial receptivity. Reprod Biomed Online. 2017; 34(4):422-8 
8. Bosch E, Valencia I, Escudero E, Crespo J, Simon C, Remohi J, et al. Premature luteinization during gonadotropin-releasing hormone antagonist cycles and its relationship with in vitro fertilization outcome. Fertil Steril. 2003:80(6):1444-9.

9. Van Vaerenbergh I, Fatemi HM, Blockeel C, Van Lommel L, In't Veld P, Schuit $F$, et al. Progesterone rise on HCG day in GnRH antagonist/rFSH stimulated cycles affects endometrial gene expression. Reprod Biomed Online. 2011; 22(3):263-71.

10. Glujovsky D, Pesce R, Fiszbajn G, Sueldo C, Hart RJ, Ciapponi A. Endometrial preparation for women undergoing embryo transfer with frozen embryos or embryos derived from donor oocytes. Cochrane Database Syst Rev. 2010(1):Cd006359.

11. Groenewoud ER, Cantineau AE, Kollen BJ, Macklon NS, Cohlen BJ. What is the optimal means of preparing the endometrium in frozen-thawed embryo transfer cycles? A systematic review and meta-analysis. Hum Reprod Update. 2017;23(2):255-61.

12. Mackens S, Santos-Ribeiro S, van de Vijver A, Racca A, Van Landuyt L, Tournaye $H$, et al. Frozen embryo transfer: a review on the optimal endometrial preparation and timing. Hum Reprod. 2017;32(11):2234-42.

13. Davar R, Janati S, Mohseni F, Khabazkhoob M, Asgari S. A comparison of the effects of transdermal estradiol and estradiol Valerate on endometrial receptivity in frozen-thawed embryo transfer cycles: a randomized clinical trial. J Reprod Infertil. 2016;17(2):97-103.

14. Shapiro DB, Pappadakis JA, Ellsworth NM, Hait HI, Nagy ZP. Progesterone replacement with vaginal gel versus i.m. injection: cycle and pregnancy outcomes in IVF patients receiving vitrified blastocysts. Hum Reprod. 2014; 29(8):1706-11.

15. Devine K, Richter KS, Widra EA, McKeeby JL. Vitrified blastocyst transfer cycles with the use of only vaginal progesterone replacement with Endometrin have inferior ongoing pregnancy rates: results from the planned interim analysis of a three-arm randomized controlled noninferiority trial. Fertil Steril. 2018;109(2):266-75.

16. Nawroth F, Ludwig M. What is the 'ideal' duration of progesterone supplementation before the transfer of cryopreserved-thawed embryos in estrogen/progesterone replacement protocols? Hum Reprod. 2005;20(5): 1127-34.

17. van de Vijver A, Polyzos NP, Van Landuyt L, Mackens S, Stoop D, Camus M, et al. What is the optimal duration of progesterone administration before transferring a vitrified-warmed cleavage stage embryo? A randomized controlled trial. Hum Reprod. 2016:31(5):1097-104.

18. Kofinas JD, Blakemore J, McCulloh DH, Grifo J. Serum progesterone levels greater than $20 \mathrm{ng} / \mathrm{dl}$ on day of embryo transfer are associated with lower live birth and higher pregnancy loss rates. J Assist Reprod Genet. 2015;32(9): 1395-9.

19. Brady PC, Kaser DJ, Ginsburg ES, Ashby RK, Missmer SA, Correia KF, et al. Serum progesterone concentration on day of embryo transfer in donor oocyte cycles. J Assist Reprod Genet. 2014;31(5):569-75.

20. Gaggiotti-Marre S, Martinez F, Coll L, Garcia S, Alvarez M, Parriego M, et al. Low serum progesterone the day prior to frozen embryo transfer of euploid embryos is associated with significant reduction in live birth rates. Gynecol Endocrinol. 2018:1-4

21. Labarta E, Mariani G, Holtmann N, Celada P, Remohi J, Bosch E. Low serum progesterone on the day of embryo transfer is associated with a diminished ongoing pregnancy rate in oocyte donation cycles after artificial endometrial preparation: a prospective study. Hum Reprod. 2017;32(12): 2437-42.

22. Niu Z, Feng $Y$, Sun $Y$, Zhang A, Zhang H. Estrogen level monitoring in artificial frozen-thawed embryo transfer cycles using step-up regime without pituitary suppression: is it necessary? J Exp Clin Assist Reprod. 2008;5:4

23. Rittenberg V, Seshadri S, Sunkara SK, Sobaleva S, Oteng-Ntim E, El-Toukhy T. Effect of body mass index on IVF treatment outcome: an updated systematic review and meta-analysis. Reprod Biomed Online. 2011;23(4): 421-39

24. Shah DK, Missmer SA, Berry KF, Racowsky C, Ginsburg ES. Effect of obesity on oocyte and embryo quality in women undergoing in vitro fertilization. Obstet Gynecol. 2011;118(1):63-70.

25. Tremellen K, Pearce K, Zander-Fox D. Increased miscarriage of euploid pregnancies in obese women undergoing cryopreserved embryo transfer. Reprod Biomed Online. 2017;34(1):90-7.

26. Zhang D, Zhu Y, Gao H, Zhou B, Zhang R, Wang T, et al. Overweight and obesity negatively affect the outcomes of ovarian stimulation and in vitro fertilisation: a cohort study of 2628 Chinese women. Gynecol Endocrinol. 2010;26(5):325-32.

27. Criteria for number of embryos to transfer: a committee opinion. Fertil Steril. 2013;99(1):44-6.

28. Guidance on the limits to the number of embryos to transfer: a committee opinion. Fertil Steril. 2017;107(4):901-3.

\section{Publisher's Note}

Springer Nature remains neutral with regard to jurisdictional claims in published maps and institutional affiliations.
Ready to submit your research? Choose BMC and benefit from:

- fast, convenient online submission

- thorough peer review by experienced researchers in your field

- rapid publication on acceptance

- support for research data, including large and complex data types

- gold Open Access which fosters wider collaboration and increased citations

- maximum visibility for your research: over $100 \mathrm{M}$ website views per year

At BMC, research is always in progress.

Learn more biomedcentral.com/submissions 